\title{
Application of Commutator Theorems to the Integration of Representations of Lie Algebras and Commutation Relations*
}

\section{J. Fröhlich $\star \star$}

Department of Mathematics, Princeton University, Princeton, NJ 08540, USA

\begin{abstract}
Sufficient conditions on unbounded, symmetric operators $A$ and which imply that$$
\exp (i t A) \exp (i s B) \exp (-i t A)
$$

satisfies the well known "multiple commutator" formula are derived. Th formula is then applied to prove new necessary and sufficient conditions for th integrability of representations of Lie algebras and canonical commutatic relations and the commutativity of the spectral projections of two commutin unbounded, self-adjoint operators. A classic theorem of Nelson's is obtained : a corollary. Our results are useful in relativistic quantum field theory.
\end{abstract}

\section{Introduction}

In this note we discuss sufficient conditions for the multiple commutator formu

$$
e^{i t A} e^{i s B} e^{-i t A}=\exp \text { is }\left\{B+\sum_{n=1}^{\infty} \frac{(i t)^{n}}{n !} \operatorname{ad}^{n} A(B)\right\},
$$

to hold. Here $A$ and $B$ are unbounded operators and, formally,

$$
\begin{aligned}
\operatorname{ad} A(B) & =[A, B], \\
\operatorname{ad}^{n} A(B) & =\left[A, \operatorname{ad}^{n-1} A(B)\right] .
\end{aligned}
$$

Our results have applications in group theory and quantum field theory.

They are a direct outgrowth of recent work of Driessler and the author [ 2 concerning the Haag-Kastler axioms [12] in relativistic quantum field theory and subsequent alternate proof of the main result of [2] due to Glimm and Jaffe [3

The main result of $[2,3]$, a sufficient condition for the bounded functions of tw unbounded, symmetric operators $A$ and $B$ to commute, is a special case of th results proven in the following sections.

* Research supported in part by the US National Science Foundation under Grant MPS 75-118t

$\star \star$ A Sloan Foundation Fellow 
The basic strategy for a proof of (1.1) is to find a self-adjoint operator $N \geqq 1$ in terms of which $A, B$ and $i^{n}$ ad $^{n} A(B), n=1,2, \ldots$, can be dominated in the sense of the commutator theorem of [4]; see also [6]. (Different forms of the commutator theorem may be found in [8].)

\section{The Main Results}

We start with describing the general set-up and recalling the commutator theorem.

Let $\mathscr{H}$ be a separable Hilbert space, and $N$ a positive, self-adjoint operator on $\mathscr{H}$ satisfying

$$
N \geqq 1 \text {. }
$$

We let $\mathscr{H}_{n}$ be (the completion of) $D\left(N^{n / 2}\right)$ in the norm

$$
\|\psi\|_{n}=\left\|N^{n / 2} \psi\right\|, \pm n=1,2,3 \ldots,
$$

and $\mathscr{L}\left(\mathscr{H}_{n}, \mathscr{H}_{m}\right)$ the bounded operators from $\mathscr{H}_{n}$ to $\mathscr{H}_{m}$.

The domain of an operator $C$ is denoted $D(C)$, and a subspace $\mathscr{D} C \mathscr{H}$ is called a core for $C$ if

$$
C=(C \uparrow \mathscr{D})^{-} \text {. }
$$

Here $C \uparrow \mathscr{D}$ denotes the restriction of $C$ to the subspace $\mathscr{D}$ and ( $)^{-}$the closure of ( ).

We assume that $A$ is a symmetric operator in $\mathscr{L}\left(\mathscr{H}_{1}, \mathscr{H}_{-1}\right)$; i. e., on some form core for $N\left(\equiv\right.$ core for $\left.N^{1 / 2}\right)$,

$$
\pm A \leqq K_{1} N
$$

for some finite constant $K_{1}$ (in the quadratic form sense). Then

$$
\dot{A} \equiv i[N, A]
$$

is defined as an element of $\mathscr{L}\left(\mathscr{H}_{3}, \mathscr{H}_{-3}\right)$ in the obvious way; see e.g. [8].

Theorem 0. Let $A$ be as above and assume, in addition, that $\dot{A} \in \mathscr{L}\left(\mathscr{H}_{1}, \mathscr{H}_{-1}\right)$, i.e., on a form core for $N$,

$$
\pm \dot{A} \leqq K_{1} N \text {. }
$$

Then $A$ determines a densely defined, symmetric operator - also denoted $A$-on $\mathscr{H}$ with

$$
D(N) \leqq D(A),\|A \psi\| \leqq k_{1}\|N \psi\|, \quad \text { with } \quad k_{1} \leqq 2^{1 / 2} K_{1}
$$

for all $\psi \in D(N)$, and

$A$ is essentially self-adjoint on any core for $N$.

Remark. This is the commutator theorem of [4], stated in a form due to [6].

In the applications a slightly different form of the commutator theorem is sometimes more useful. 
Theorem $\mathbf{0}^{\prime}$. Let $A$ be a symmetric operator on $\mathscr{H}$ with the properties that

$D(A)$ contains a core $\mathscr{D}$ for $N$,

$$
\begin{aligned}
& \|A \psi\| \leqq k_{1}\|N \psi\|, \quad \text { and } \\
& \pm i\{(N \psi, A \psi)-(A \psi, N \psi)\} \leqq K_{1}\left\|N^{1 / 2} \psi\right\|^{2}
\end{aligned}
$$

for all $\psi \in \mathscr{D}$.

Then the conclusions of Theorem 0 remain true.

For proofs of Theorems 0 and $0^{\prime}$, related results and references see [8], (Theorems X.36, X.36', and X.37).

We now state our main results.

Theorem $\mathbf{1}_{\mathbf{M}}$. Let $A, \dot{A}, B$, and $\left\{C_{n}\right\}_{n=0}^{M}$ be operators in $\mathscr{L}\left(\mathscr{H}_{1}, \mathscr{H}_{-1}\right)$ satisfying the hypotheses of Theorems 0 or $0^{\prime}$. Assume that $C_{0}=B$, and

$$
C_{n}=i\left[A, C_{n-1}\right] \text {, }
$$

in $\mathscr{L}\left(\mathscr{H}_{2}, \mathscr{H}_{-2}\right)$ (i.e., weakly on $\left.D(N) \times D(N)\right)$, for all $n=1, \ldots, M$. Then

$$
e^{i t A} e^{i s B} e^{-i t A}=e^{i s B_{t}},
$$

with

$$
\begin{aligned}
B_{t}= & {\left[\left\{B+\sum_{n=1}^{M-1} \frac{t^{n}}{n !} C_{n}\right.\right.} \\
& \left.\left.+\int_{0}^{t} d t_{1} \ldots \int_{0}^{t_{M}-1} d t_{M} e^{i t_{M} A} C_{M} e^{-i t_{M} A}\right\} \uparrow D(N)\right]^{-} .
\end{aligned}
$$

Theorem $1_{\infty}$. Let $A, \dot{A}, B$ and $\left\{C_{n}\right\}_{n=0}^{\infty}$ be as in the hypotheses of Theorem $1_{\mathrm{M}}$, (for $M$ $=\infty)$, and assume, in addition, that there is some finite constant $K_{2}$ such that, on a form core for $N$

$$
\left.\begin{array}{l} 
\pm C_{n} \leqq K_{2}^{n} n ! N \\
\pm \dot{C}_{n} \leqq K_{2}^{n} n ! N
\end{array}\right\}
$$

for all $n=1,2,3, \ldots$.

Then, for $|t|<K_{2}^{-1}$,

$$
\lim _{M \rightarrow \infty}\left\{B+\sum_{n=1}^{M} \frac{t^{n}}{n !} C_{n}\right\} \uparrow D(N) \text { exists, }
$$

has a self-adjoint closure, denoted $B_{t}$, and

$$
e^{i t A} e^{i s B} e^{-i t A}=e^{i s B_{t}}
$$

Remarks. 1) One may also denote $C_{n}$ by $i^{n} \operatorname{ad}^{n} A(B)$; see (1.1) and (1.2).

2) A generalization of Theorems $1_{M}$ and $1_{\infty}$ which may be useful in various applications is presented in an Appendix; see Lemma A.1 and Theorem A.2.

3) Theorem $1_{M}$ contains as special cases sufficient conditions for the commutativity of the bounded functions of two unbounded, self-adjoint operators 
(equivalent to the ones found in [2]) and for the integrability of the canonical commutation relations; see Sections 6 and 7. Theorem $1_{\infty}$ is useful for the integration of equations of motion in the "Heisenberg picture".

\section{Preliminaries: Invariance of Operator Domains}

Here we discuss some results concerning the invariance of the domain $D(N)$ of $N$ under certain unitary groups. They represent a slight elaboration of results of Glimm and Jaffe [3] and may be of some interest in their own right. See also Lemma A.1 in the Appendix.

We let $Q(N)=D\left(N^{1 / 2}\right)$ denote the quadratic form domain of $N$.

The main result of this section is

Lemma 2. Let $A$ and $\dot{A}$ satisfy the hypotheses of Theorem 0 or $0^{\prime}$. Then

1) $e^{i t A} Q(N) \cong Q(N)$, and, for all $\psi \in Q(N)$,

$\left\|N^{1 / 2} e^{i t A} \psi\right\| \leqq e^{(1 / 2) K_{1}|t|}\left\|N^{1 / 2} \psi\right\|$;

2) $e^{i t A} D(N) \leqq D(N)$, and, for all $\psi \in D(N)$,

$\left\|N e^{i t A} \psi\right\| \leqq e^{k_{1}|t|}\|N \psi\|$;

3) $e^{i t A} D\left(N^{\alpha}\right)=D\left(N^{\alpha}\right)$, for $\alpha=1 / 2,1$.

Remark. 3) is an immediate consequence of 1) and 2).

Proof. We first prove a simpler version of Lemma 2.

Definition. For $\lambda \geqq 0$ we set

$$
R(\lambda) \equiv(N+\lambda)^{-1}, \quad N_{\lambda} \equiv \lambda^{2} R(\lambda) N R(\lambda),
$$

and

$$
\stackrel{(\cdot)}{A} \equiv \lambda^{2} R(\lambda) \stackrel{(\cdot)}{A} R(\lambda) .
$$

Here $\stackrel{(\cdot)}{A}$ denotes $A$ or $\dot{A}$.

Using the self-adjointness and strict positivity of $N$ we get

$$
N_{\lambda}^{\alpha} \leqq N^{\alpha}, \text { for all } \alpha \geqq 1,
$$

and since $A$ and $\dot{A}$ satisfy (2.4), for some $K_{1}<\infty$

$$
\|\stackrel{(\cdot)}{A}\| \geqq \leqq \lambda K_{1} \text {. }
$$

Application of (2.5) yields

$$
\left\|N^{\alpha} \stackrel{(\cdot)}{A_{\lambda}}\right\| \leqq \lambda^{1+\alpha} k_{1} \text {, for } \alpha \in[0,1] .
$$

Lemma $2_{\lambda}$. Lemma 2 holds with $A_{\lambda}$ replacing $A$ and constants $K_{1}$ and $k_{1}$ independent of $\lambda$. 
Proof'. Using (3.4) and (3.5) and the power series expansion of $e^{i t A_{\lambda}}$ (convergent for all $|t|<\infty, \lambda<\infty)$ we obtain:

$$
e^{i t A_{\lambda}} Q(N)=Q(N), \quad e^{i t A_{\lambda}} D(N)=D(N) .
$$

For $\psi \in Q(N)$, set

Then

$$
F_{\lambda}(t)=\left(e^{i t A_{\lambda}} \psi, N e^{i t A_{\lambda}} \psi\right) .
$$

$$
\begin{aligned}
d F_{\lambda}(t) / d t & =\left(e^{i t A_{\lambda}} \psi, \dot{A}_{\lambda} e^{i t A_{\lambda}} \psi\right) \\
& \leqq K_{1}\left(e^{i t A_{\lambda}} \psi, N_{\lambda} e^{i t A_{\lambda}} \psi\right) \\
& \leqq K_{1} F_{\lambda}(t),
\end{aligned}
$$

and we have used (3.2), (2.4), and (3.3). Hence

$$
F_{\lambda}(t) \leqq e^{K_{1}|t|} F_{\lambda}(0)=e^{K_{1}|t|}\left\|N^{1 / 2} \psi\right\|^{2}
$$

which proves Lemma $2_{\lambda},(1)$. Next, let $\psi \in D(N)$ and set

$$
G_{\lambda}(t)=\left(N e^{i t A_{\lambda}}, N e^{i t A_{\lambda}} \psi\right) .
$$

Then

$$
\begin{aligned}
d G_{\lambda}(t) / d t & =\left(N e^{i t A_{\lambda}} \psi, \dot{A}_{\lambda} e^{i t A_{\lambda}} \psi\right)+\left(\dot{A}_{\lambda} e^{i t A_{\lambda}} \psi, N e^{i t A_{\lambda}} \psi\right) \\
& \leqq 2\left\|N e^{i t A_{\lambda}} \psi\right\|\left\|\dot{A}_{\lambda} e^{i t A_{\lambda}} \psi\right\| \\
& \leqq 2\left\|N e^{i t A_{\lambda}} \psi\right\|\|\lambda R(\lambda)\|\left\|\dot{A} \lambda R(\lambda) e^{i t A_{\lambda}} \psi\right\| \\
& \leqq 2 k_{1}\|\lambda R(\lambda)\|^{2}\left\|N e^{i t A_{\lambda}} \psi\right\|^{2} \leqq 2 k_{1} G_{\lambda}(t),
\end{aligned}
$$

and we have used (3.2), (2.5), and (3.3). Therefore

$$
\begin{aligned}
G_{\lambda}(t) & \leqq e^{2 k_{1}|t|} G_{\lambda}(0) \\
& =e^{2 k_{1}|t|}\|N \psi\|^{2},
\end{aligned}
$$

which proves Lemma $2_{\lambda},(2)$. Lemma $2_{\lambda},(3)$ is statement (3.6). Hence the proof is complete.

As a corollary to Lemma $2_{\lambda},(2)$ we note that

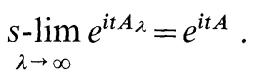

Since $\left\{e^{i t A_{\lambda}}: \lambda \geqq 0\right\}$ and $e^{i t A}$ are unitary operators, it suffices to prove weak convergence on a dense set. For $\varphi$ and $\psi$ in $D(N)$,

$$
\begin{aligned}
& \left(\varphi,\left\{e^{i t A}-e^{i t A_{\lambda}}\right\} \psi\right) \\
& =i \int_{0}^{1} d s\left(\{1-\lambda R(\lambda)\} e^{-i s A} \varphi,\left(A N^{-1}\right) \lambda R(\lambda) N e^{i(t-s) A_{\lambda}} \psi\right) \\
& \quad+i \int_{0}^{1} d s\left(\{1-\lambda R(\lambda)\} e^{-i s A} A \varphi, e^{i(t-s) A_{\lambda}} \psi\right) .
\end{aligned}
$$

Since $\{1-\lambda R(\lambda)\}$ tends to 0 strongly, $A N^{-1}$ is bounded by $(2.5),\|\lambda R(\lambda)\| \leqq 1$ and $\left\|N e^{i(t-s) A} \psi\right\| \|$ is bounded uniformly in $\lambda$, the integrands tend to 0 , for all $s$. Since the 
integrands are bounded uniformly in $s$ and $\lambda$, the r.h.s. of (3.12) tends to 0 by the Lebesgue dominated convergence theorem.

We summarize:

i) For $\psi \in D\left(N^{1 / 2}\right),\left\|N^{1 / 2} e^{i t A_{\lambda}} \psi\right\| \leqq e^{(1 / 2) K_{1}|t|}\left\|N^{1 / 2} \psi\right\|$;

ii) for $\psi \in D(N),\left\|N e^{i t A_{\lambda}} \psi\right\| \leqq e^{k_{1}|t|}\|N \psi\|$, uniformly in $\lambda$, and

iii) Equation (3.11).

We now complete the proof of Lemma 2.

Combination of i) - iii) with the spectral theorem applied to $N$ immediately gives Lemma 2,1) and 2). To prove 3), note that by 1) and 2)

$e^{ \pm i t A} D\left(N^{\alpha}\right) \subseteq D\left(N^{\alpha}\right), \quad$ for all $|t|<\infty$

and $\alpha=1 / 2,1$. Thus

$$
\begin{aligned}
D\left(N^{\alpha}\right) & =e^{i t A}\left\{e^{-i t A} D\left(N^{\alpha}\right)\right\} \\
& \cong e^{i t A} D\left(N^{\alpha}\right)
\end{aligned}
$$

hence $D\left(N^{\alpha}\right)=e^{i t A} D\left(N^{\alpha}\right)$, for all $|t|<\infty$. Q.E.D.

Remarks. Lemma 2,2) and the trick of using a differential inequality for $G_{\lambda}(t)$ are due to [3]; (we have applied it in a slightly different form, and an extension is presented in an Appendix: Proof of sufficient conditions for

$$
\left.e^{i t A} D\left(N^{\alpha}\right)=D\left(N^{\alpha}\right), \alpha \in(-\infty, \infty)\right) \text {. }
$$

We note that, by Lemma 2, 3) and Theorem $0,\left(0^{\prime}\right)$

$e^{-i t A} D(N)$ is a core for $B$

if $B$ satisfies the hypotheses of Theorem $0,\left(0^{\prime}\right)$.

Lemma 2 may be summarized as follows : $\left\{e^{i t A}\right\}$ determines unique, exponentially bounded one parameter groups on the spaces $\mathscr{H}_{n}$, for $n=-2,-1,0,1,2$.

\section{Proofs of Theorems $1_{M}$ and $1_{\infty}$}

Let $A, B$, and $\left\{C_{n}\right\}$ satisfy the hypotheses of Theorem $1_{\mathrm{M}}$ or $1_{\infty}$. By Theorem $0, B$ is essentially self-adjoint on $D(N)$. Its closure is also denoted $B$. Let

$$
B=\int \lambda d E(\lambda)
$$

be the spectral decomposition of $B$. We set

$$
\left.\begin{array}{rl}
B_{t} & =\int \lambda d E_{t}(\lambda), \text { with } \\
E_{t}(\cdot) & =e^{i t A} E(\cdot) e^{-i t A} .
\end{array}\right\}
$$

Then we conclude from (3.14) (by the fundamental criterion) that $D(N)$ is a core for $B_{t}$, i.e., $B_{t}$ is essentially self-adjoint on $D(N)$. By Lemma 2, 2) and (4.1)

$$
B_{t}=e^{i t A} B e^{-i t A} \text {, on } D(N) \text {. }
$$


Let $\psi$ and $\theta$ be in $D(N)$. Then, using Lemma 2, 2), the hypotheses on $B$ and $C_{1}$, see (2.7), and Theorem $0,(2.5)$, we obtain

$$
\begin{aligned}
d\left(\psi, B_{t} \theta\right) / d t= & i\left\{\left(A e^{-i t A} \psi, B e^{-i t A} \theta\right)\right. \\
& \left.-\left(B e^{-i t A} \psi, A e^{-i t A} \theta\right)\right\} \\
= & \left(\psi, e^{i t A} C_{1} e^{-i t A} \theta\right)
\end{aligned}
$$

and

$$
\begin{aligned}
\left|d\left(\psi, B_{t} \theta\right) / d t\right| & \leqq k_{2}\|\psi\|\left\|N e^{-i t A} \theta\right\| \\
& \leqq k_{2} e^{k_{1}|t|}\|\psi\|\|N \theta\|
\end{aligned}
$$

Thus, for all $\theta \in D(N)$,

$$
d B_{t} \theta / d t=e^{i t A} C_{1} e^{-i t A} \theta,
$$

and hence

$$
\left(A_{1}\right) B_{t} \theta=B \theta+\int_{0}^{t} d s e^{i s A} C_{1} e^{-i s A} \theta .
$$

Since $D(N)$ is a core for $B_{t}$, we conclude:

$$
B_{t}=\left[\left\{B+\int_{0}^{t} d s e^{i s A} C_{1} e^{-i s A}\right\}\lceil D(N)]^{-} .\right.
$$

We now proceed by induction: Assume

$$
\begin{aligned}
\left(A_{n}\right) B_{t}= & {\left[\left\{B+\sum_{m=1}^{n-1} \frac{t^{m}}{m !} C_{m}\right.\right.} \\
& \left.+\int_{0}^{t} d t_{1} \ldots \int_{0}^{t_{n-1}} d t_{n} e^{i t_{n} A} C_{n} e^{-i t_{n} A}\right\}\lceil D(N)]^{-} .
\end{aligned}
$$

By hypothesis on $\left\{C_{m}\right\}_{m=0}^{\infty}$ (see Theorems $1_{\mathrm{M}}, 1_{\infty}$ ), Theorem 0 and Lemma 2, 2), $D(N)$ is contained in the domain of

$$
e^{i s A} C_{m} e^{-i s A}, \text { for all } m<\infty
$$

As in the proof of $\left(A_{1}\right)$ we show that

$$
e^{i t_{n} A} C_{n} e^{-i t_{n} A}=C_{n}+\int_{0}^{t_{n}} d t_{n+1} e^{i t_{n+1} A} C_{n+1} e^{-i t_{n+1} A}
$$

on $D(N)$; [just replace $B$ by $C_{n}, C_{1}$ by $C_{n+1}, t$ by $t_{n}$ and use (4.4)].

Inserting this equation into $\left(A_{n}\right)$ and using again that $D(N)$ is a core for $B_{t}$, we immediately obtain $\left(A_{n+1}\right)$.

This completes the proof of Theorem $1_{\mathrm{M}}$.

The proof of Theorem $1_{\infty}$ is now easy: 
Using inequalities (2.9) (see Theorem $1_{\infty}$ ), (2.5) (see Theorem 0) and Lemma 2, 2) we obtain the estimate

$$
\begin{aligned}
& \left\|\int_{0}^{t} d t_{1} \ldots \int_{0}^{t_{n-1}} d t_{n} e^{i t_{n} A} C_{n} e^{-i t_{n} A} \theta\right\| \\
& \quad \leqq 2^{1 / 2} K_{2}^{n} n ! \int_{0}^{|t|} d t_{1} \ldots \int_{0}^{t_{n}-1} d t_{n}\left\|N e^{-i t_{n} A} \theta\right\| \\
& \quad \leqq 2^{1 / 2}\left(K_{2}|t|\right)^{n} e^{k_{1}|t|}\|N \theta\|,
\end{aligned}
$$

for all $\theta \in D(N)$.

For $|t|<K_{2}^{-1}$, the r.h.s. of (4.5) tends to 0 , as $n \rightarrow \infty$, and this gives the first part of Theorem $1_{\infty}$.

The second part then follows by using once more that $D(N)$ is a core for $B_{t}$.

In the following sections we indicate some applications and in the Appendix an extension of Theorems $1_{M}$ and $1_{\infty}$.

\section{An Application to Lie Groups}

\subsection{Integration of Representations of Lie Algebras}

Let $G$ be a simply connected Lie group with Lie algebra $\left(\mathfrak{b} ;\right.$ let $\left\{\xi_{1}, \ldots, \xi_{n}\right\}$ be a basis for $\mathfrak{G}$ and $\left\{c_{i j k}\right\}$ the structure constants. We consider a representation $\pi$ of $\mathfrak{G}$ on a Hilbert space $\mathscr{H}$ and define

$$
X_{j}=i \pi\left(\xi_{j}\right), \quad j=1, \ldots, n .
$$

Furthermore $N \geqq 1$ is some self-adjoint operator on $\mathscr{H}$.

The following result gives new sufficient conditions for the integrability of $\pi(\mathfrak{b})$, different from the classic ones found by Nelson in [7] (and extended e.g., in [10]).

Theorem 3. Assume that $X_{j}$ and $\dot{X}_{j}$ satisfy the hypotheses of Theorem 0 or $0^{\prime}$, for all $j$ $=1, \ldots, n$, and that there is a core $\mathscr{D}$ for $N$ such that

$$
\left[X_{l}, X_{j}\right]=i \sum_{k=1}^{n} c_{l j k} X_{k}
$$

weakly on $\mathscr{D} \times \mathscr{D}$.

Then $\pi(\mathfrak{6})$ is the differential of a continuous unitary representation $\pi^{\prime}$ of $G$ on $\mathscr{H}$ (i.e., the representation $\pi$ of $\mathbb{5}$ on $\mathscr{H}$ can be integrated to a representation $\pi^{\prime}$ of $G$ on $\mathscr{H})$.

Proof. Let $\alpha_{1}, \ldots, \alpha_{n}$ be arbitrary real numbers. By Theorem 0 (or $0^{\prime}$ )

$$
D\left(\sum_{j=1}^{n} \alpha_{j} X_{j}\right) \supseteqq D(N),
$$

and

$$
\sum_{j=1}^{n} \alpha_{j} X_{j}
$$


is essentially self-adjoint on $D(N)$, for all $j=1, \ldots, n$. Using $(2.5)$ for $X_{k}, k=1, \ldots, n$, and (5.2) we obtain

$$
\left[X_{l}, X_{j}\right]=i \sum_{k=1}^{n} c_{l j k} X_{k}, \quad \text { weakly on } \quad D(N) \times D(N) .
$$

By Lemma 2,3)

$$
\exp \left(i t \sum_{j=1}^{n} \alpha_{j} X_{j}\right) D(N)=D(N),
$$

for all $|t|<\infty$.

Since $\mathfrak{5}$ is the Lie algebra of $G$, there is some open neighborhood $U$ of $0 \in \mathfrak{F}$ which is mapped diffeomorphically onto an open neighborhood $W$ of the identity $e \in G$ by the exponential mapping. Thus, for $g \in W$, there exists some

$$
\xi=\sum_{j=1}^{n} \alpha_{j} \xi_{j} \in U \subset \mathfrak{G}
$$

with

$$
g=e^{\zeta} .
$$

We define

$$
\pi^{\prime}(g)=e^{-i X},
$$

where

$$
X=\sum_{j=1}^{n} \alpha_{j} X_{j} .
$$

Suppose now that $g, \hat{g}$ and $g \cdot \hat{g}$ are in $W$. We must show that

$$
\pi^{\prime}(g \hat{g})=\pi^{\prime}(g) \pi^{\prime}(\hat{g}) .
$$

Without loss of generality we may assume that, for all $t \in[0,1], e^{t \xi}$ and $e^{t \xi} \hat{g}$ are in $W$. Then

$$
e^{t \xi}=e^{t \sum_{j=1}^{n} \alpha_{j} \xi_{j}}
$$

and

$$
e^{t \xi} \hat{g}=e^{\sum_{j=1}^{n} \hat{\alpha}_{j}(t) \xi_{j}},
$$

where $\hat{\alpha}_{j}(t)$ is continuously differentiable in $t$ in some neighborhood of $[0,1]$, for all $j=1, \ldots, n$.

In order to prove (5.6) we now compare

$$
F(t)=\pi^{\prime}\left(e^{t \xi} \hat{g}\right) \psi
$$

with

$$
\pi^{\prime}\left(e^{t \xi}\right) \pi^{\prime}(\hat{g}) \psi=e^{-i t x} F(0),
$$

where $\psi$ is an arbitrary vector in $D(N)$. 
From (5.7) and (5.8) we know that

$$
F(t)=e^{-i Y(t)} \psi
$$

where

$$
Y(t) \equiv \sum_{j=1}^{n} \hat{\alpha}_{j}(t) X_{j}
$$

is essentially self-adjoint on $D(N)$. We set

$$
Y^{\prime}(t)=\sum_{j=1}^{n} \frac{d}{d t} \hat{\alpha}_{t}(t) X_{j}
$$

Then $Y^{\prime}(t)$ is essentially self-adjoint on $D(N)$ and by Theorem 0 (resp. $0^{\prime}$ )

$$
\left\|Y^{\prime}(t) \varphi\right\| \leqq k_{3}\|N \varphi\|,
$$

uniformly in $t \in[0,1]$; moreover

$$
\left\|N e^{-i s Y(t)} \varphi\right\| \leqq k_{4}\|N \varphi\|,
$$

uniformly in $s \in[0,1], t \in[0,1]$, for all $\varphi \in D(N)$.

From the formula

$$
e^{i A}-e^{i B}=\left(i \int_{0}^{1} d s e^{i s A}(A-B) e^{i(1-s) B}\right)^{-}
$$

and the above estimates we conclude that $e^{-i s Y(t)}$ is continuous in $t$ and that $F(t)$ is differentiable, with the following derivative:

$$
\begin{aligned}
\frac{d}{d t} F(t) & =-i \int_{0}^{1} d s e^{-i s Y(t)} Y^{\prime}(t) e^{-i(1-s) Y(t)} \psi \\
& =-i \int_{0}^{1} d s e^{-i s Y(t)} Y^{\prime}(t) e^{i s Y(t)} F(t) .
\end{aligned}
$$

The hypotheses of Theorem 3 permit us to apply the multiple commutator formula proven in Theorem $1_{\infty}$ to compute

$$
Y(s, t) \equiv e^{-i s Y(t)} Y^{\prime}(t) e^{i s Y(t)}
$$

on $D(N)$, and then integrate over $s$ : From Theorem 0 (resp. $0^{\prime}$ ) and (3.14) we know that $Y(s, t)$ and $\int_{0}^{1} d s Y(s, t)$ are essentially self-adjoint on $D(N)$, so that it suffices to
identify

$$
\int_{0}^{1} d s Y(s, t) \uparrow D(N)
$$

We now claim that

$$
\int_{0}^{1} d s Y(s, t) \uparrow D(N)=X \uparrow D(N) \text {. }
$$


Using the hypotheses of Theorem 3, Theorem 0 (resp. $0^{\prime}$ ) and Lemma 2 we easily derive the required estimates which guarantee that we may apply Theorem $1_{\infty}$. This theorem then tells us that (5.11) holds if it holds formally, i.e., if

$$
\int_{0}^{1} d s e^{s \sum_{j=1}^{n} \hat{\alpha}_{j}(t) \xi_{j}}\left(\sum_{j=1}^{n} \frac{d}{d t} \hat{\alpha}_{j}(t) \xi_{j}\right) e^{-s \sum_{j}^{n} \hat{\alpha}_{j}(t) \xi_{j}}=\xi
$$

holds as an equation between two elements of $\mathfrak{6}$. This, however, is obvious.

From (5.10) and (5.11) we conclude that $F(t)$ satisfies

$$
d F(t) / d t=-i X F(t) \text {. }
$$

Since $F(t) \in D(N)$, for all $t \in[0,1]$, and $D(N)$ is a core for $X$, and since $F(0)=\pi^{\prime}(\hat{g}) \psi$, we conclude that $F(1)=e^{-i X} F(0)=\pi^{\prime}\left(e^{\xi}\right) \pi^{\prime}(\hat{g}) \psi$.

The proof of (5.6) is now complete, because $D(N)$ is dense in $\mathscr{H}$. Q.E.D.

We note that the idea of using a differential equation and Theorem $1_{\infty}$ to prove $F(t)=e^{-i t X} F(0)$, for all $t \in[0,1]$, avoids the use of the Baker-Hausdorff-Campbell formula in the proof of $(5.6)$ (see also $[7,10]$ ).

From Theorem 3 we immediately obtain the following classic result of Nelson [7].

Corollary 4. If

$$
N^{\prime}=\sum_{j=1}^{n} X_{j}^{2}+1
$$

is essentially self-adjoint on a dense domain $\mathscr{D} \subset \mathscr{H}$ then

$$
N=\overline{N^{\prime} \uparrow \mathscr{D}}
$$

has the properties of the operator $N$ of Theorem 3 , and all the conclusions of Theorem 3 hold.

Remark. Corollary 4 and the results of [7] show that the converse of Theorem 3 is true, too.

\subsection{A Trivial Application to the Rotation Group}

Here we consider $G=\operatorname{SU}(2)$.

Let $\boldsymbol{x}$ denote the vectors in $R^{3}$, and

$$
\mathscr{H}=L^{2}\left(R^{3}\right), \quad \boldsymbol{p}=-i\left(\frac{\partial}{\partial x_{1}}, \frac{\partial}{\partial x_{2}}, \frac{\partial}{\partial x_{3}}\right),
$$

with $x_{i}$ the $i^{\text {th }}$ component of $\boldsymbol{x}$. Define

$$
\boldsymbol{L}=\left(L_{1}, L_{2}, L_{3}\right)=\boldsymbol{x} \wedge \boldsymbol{p} ; \quad(\boldsymbol{L}, \boldsymbol{a})=\sum_{j=1}^{3} L_{j} a_{j} .
$$

We set $N=\frac{1}{2}\left(x^{2}+p^{2}+1\right)$ 
It is a well known exercise to show that the hypotheses of Theorem 3 are fulfilled for this choice of $N$ and $X_{j} \doteq L_{j}, j=1,2,3$; e.g.

$$
\begin{aligned}
\pm(\psi,(\boldsymbol{L}, \boldsymbol{a}) \psi) & \leqq|\boldsymbol{a}|\||\boldsymbol{x}| \psi\| \cdot\||\boldsymbol{p}| \psi\| \\
& \leqq \frac{|\boldsymbol{a}|}{2}\left\{\||\boldsymbol{x}| \psi\|^{2}+\||\boldsymbol{p}| \psi\|^{2}\right\} \\
& \leqq|\boldsymbol{a}|(\psi, N \psi),
\end{aligned}
$$

and

$$
(\boldsymbol{L}, \boldsymbol{a})=0, \quad \text { on } \quad \mathscr{L}\left(\mathscr{H}_{3}, \mathscr{H}_{-3}\right) .
$$

Thus

$$
\left\{e^{i(\boldsymbol{L}, \boldsymbol{a})}: \boldsymbol{a} \in R^{3}\right\}
$$

is a continuous unitary representation of $\mathrm{SU}(2)$, by Theorem 3 . If we know, a priori, that $(\boldsymbol{L}, \boldsymbol{L})$ is essentially self-adjoint on some domain $\mathscr{D}$ we may re-define

$$
N=\{\overline{(\boldsymbol{L}, \boldsymbol{L}+1\}\lceil\mathscr{D}}
$$

and arrive at the same conclusions.

\section{Commutativity of Unbounded Operators and an Application to Relativistic Quantum Field Theory}

Theorem 5 [2]. Let $A, \dot{A}$, and $B$ satisfy the hypotheses of Theorem 0 (or $\left.0^{\prime}\right)$, and $[A, B]=0$, weakly on $D(N) \times D(N)$. Then all bounded functions of $A$ and $B$ commute.

Proof. An immediate consequence of Theorem $1_{1}$.

Remark. If there is some domain $\mathscr{D}$ dense in $\mathscr{H}$ such that

$$
\left(A^{2}+B^{2}\right) \uparrow \mathscr{D}
$$

is essentially self-adjoint then the hypotheses of Theorem 5 are true with

$$
N=\overline{\left(A^{2}+B^{2}+1\right) \uparrow \mathscr{D}} \text {. }
$$

[On the other hand: If all bounded functions of $A$ and $B$ commute then $\mathscr{D} \equiv D\left(A^{2}\right) \cap D\left(B^{2}\right)$ is obviously dense and $\left(A^{2}+B^{2}+1\right) \uparrow \mathscr{D}$ is self-adjoint].

Application to Quantum Field Theory [2,3]. Let $\mathscr{H}, H,\left\{\varphi(f): f \in \mathscr{S}_{\text {real }}\left(R^{d}\right)\right\}$ denote the Hilbert space, the Hamiltonian, the quantum fields, respectively, of a quantum field theory satisfying all Wightman axioms [11] and, in addition,

$$
\pm \varphi(f) \leqq|f|(H+1)
$$

for some norm $|\cdot|$ continuous on Schwartz space. Then the bounded functions of $\left\{\varphi(f): f \in \mathscr{S}_{\text {real }}\left(R^{d}\right)\right\}$ generate a net of local von Neumann algebras satisfying all Haag-Kastler axioms, [12].

Proof. Set

$$
N=H+1 \text {. }
$$


If $f$ and $g$ are two test functions with space-like separated support set

$$
A=\varphi(f), \quad B=\varphi(g) .
$$

Then $A, B$, and $N$ satisfy the hypotheses of Theorem 5 ; see [2]. [This is a consequence of (6.1) and Wightman's form of locality.] For details see [2]. Q.E.D.

Let $\mathscr{D}_{W}$ be the Wightman (polynomial) domain in $\mathscr{H}$. The remark following Theorem 5 tells us that if (6.1) is replaced by the condition that $\left(\varphi(f)^{2}+\varphi(g)^{2}\right)\left\lceil\mathscr{D}_{W}\right.$ be essentially self-adjoint, for all test functions $f$ and $g$ with space-like separated supports the theory also fulfills all Haag-Kastler axioms.

It is easy to see that with $N$ as in (6.2), $A=\varphi(f), f \in \mathscr{S}_{\text {real }}\left(R^{d}\right)$, all hypotheses of Lemmas 2 and A.1 follow from (6.1). These lemmas then tell us that, for $\psi \in D\left(H^{\alpha}\right)$, (e. g., $\psi \in \mathscr{D}_{W}$ or $\psi=\Omega$, the physical vacuum)

$$
e^{i \varphi(f)} \psi \in D\left(H^{\alpha}\right),
$$

for all $\alpha=1,2,3, \ldots$.

\section{Integration of Canonical Commutation Relations}

Let $\mathscr{H}, H$, and $\left\{\varphi(f): f \in \mathscr{S}_{\text {real }}\left(R^{d-1}\right)\right\},\left\{\pi(f): f \in \mathscr{S}_{\text {real }}\left(R^{d-1}\right)\right\}$ be the Hilbert space, the Hamiltonian, the time 0 -fields and their canonically conjugate momenta, resp., of a canonical quantum field theory [1] that satisfies, in addition,

$$
\varphi(f) \leqq|f|_{1} \cdot N,
$$

with $N \equiv H+1$,

$$
\begin{aligned}
\pi(f) & =i[N, \varphi(f)], \quad \text { on } \quad \mathscr{L}\left(\mathscr{H}_{3}, \mathscr{H}_{-3}\right), \\
\pi(f) & \leqq|f|_{2} \cdot N
\end{aligned}
$$

and

$$
\pi(f)^{\cdot} \leqq|f|_{3} \cdot N,
$$

for some Schwartz space norms $|\cdot|_{1},|\cdot|_{2}$, and $|\cdot|_{3}$. Since we are dealing with a canonical field theory, we must have

$$
[\varphi(f), \pi(g)]=i(f, g),
$$

weakly on $D(N) \times D(N)$.

Then $\varphi(f)$ and $\pi(f)$ are essentially self-adjoint on $D(N)$, for all $f \in \mathscr{S}_{\text {real }}\left(R^{d-1}\right)$, and (1) $e^{i \varphi(f)} e^{i \pi(g)}=e^{i \pi(g)} e^{i \varphi(f)} e^{-i(f, g)}$,

(the Weyl relations; set $A=\varphi(f), B=\pi(g)$ and apply Theorem $1_{2}$ ).

(2) $e^{i \varphi(f)} \pi(g) e^{-i \varphi(f)}=\pi(g)-(g, f)$;

(set $A=\varphi(f), B=\pi(g)$; apply Lemma 2,2), and (7.2)). A similar equation holds with $\varphi(f) \rightarrow \pi(f), \pi(g) \rightarrow \varphi(g)$.

(3) $e^{ \pm i \varphi(f)} H e^{ \pm i \varphi(f)}=H \mp \pi(f)+\frac{1}{2}\|f\|_{2}^{2}$ 
on $D(N) ;($ set $A=\varphi(f), B=H$ and use Lemma 2, 2), (7.1), and Theorem 0). Hence $\pm \pi(f) \leqq H+\frac{1}{2}\|f\|_{2}^{2}$ on $Q(H)$.

These results are in some sense a converse to the results of Herbst, [5].

They [in particular 1) - 3)], are very useful to give an easy proof of the fact that the $P(\phi)_{2}$ quantum field models [9] define canonical quantum field theories, [4], which requires only information on Euclidean Green's functions.

Other application of Theorems $0,0^{\prime}, 1_{\mathrm{M}}, 1_{\infty}$ to canonical commutation relations (e. g., a simple proof of von Neumann's uniqueness theorem) can be worked out quite easily and are therefore not discussed here.

\section{Appendix}

In this appendix we generalize Lemma 2 of Section 3 and reformulate Theorems $1_{M}$, $1_{\infty}$. First we prove

Lemma A.1. Suppose $A, \dot{A}, \dot{A} \equiv(\dot{A}) \stackrel{\cdot(2)}{A}, \ldots, \stackrel{(|n|)}{A}$ all satisfy the hypotheses of Theorem $0,\left(\right.$ resp. $\left.0^{\prime}\right)$. Then

$$
e^{i t A} D\left(N^{n}\right)=D\left(N^{n}\right)
$$

and

$$
\left\|N^{n} e^{i t A} \psi\right\| \leqq e^{k_{3}|t|}\left\|N^{n} \psi\right\|
$$

for some finite constant $k_{3}$, all $|t|<\infty ;( \pm n=1,2,3, \ldots)$.

Proof. We may assume $n>0$. As in Section 2 we first prove a Lemma A. $1_{\lambda}$, but

$$
A_{\lambda}=\lambda^{2 n} R(\lambda)^{n} A R(\lambda)^{n} \text {. }
$$

Then

$$
A_{\lambda}^{m} e^{i t A_{\lambda}} D\left(N^{n}\right) \subseteq D\left(N^{n}\right)
$$

is obviously true, for $m=0,1,2, \ldots$.

Let $\psi \in D\left(N^{n}\right)$. By (A.2) we may define

$$
G_{\lambda}(t)=\left(N^{n} e^{-i t A_{\lambda}} \psi, N^{n} e^{-i t A_{\lambda}} \psi\right) .
$$

Then

$$
\begin{aligned}
d G_{\lambda}(t) / d t & =i\left(e^{-i t A_{\lambda}} \psi,\left[A_{\lambda}, N^{2 n}\right] e^{-i t A_{\lambda}} \psi\right) \\
& =i \sum_{k=0}^{2 n-1}\left(e^{-i t A_{\lambda}} \psi, N^{k} \dot{A}_{\lambda} N^{2 n-k-1} e^{-i t A_{\lambda}} \psi\right)
\end{aligned}
$$

is easily shown by use of (A.1), (A.2) and the hypotheses of Lemma A.1. Next one can show that

$$
\begin{aligned}
N^{k} \dot{A}_{\lambda} N^{2 n-k-1}= & N^{k+1} \dot{A}_{\lambda} N^{2 n-k-2}+i N^{k} \ddot{A}_{\lambda} N^{2 n-k-2} \\
= & N^{k+1} \dot{A}_{\lambda} N^{2 n-k-2}+i N^{k+1} \ddot{A}_{\lambda} N^{2 n-k-3} \\
& -N^{k} \dddot{A}_{\lambda} N^{2 n-k-3} \\
= & \ldots, \text { on } D\left(N^{n}\right) \times D\left(N^{n}\right) .
\end{aligned}
$$


Applying this equation repeatedly, (proceeding e.g., by induction on $k$ and on $n$ ), using the hypotheses on $A, \dot{A}, \ldots, A$, Theorem 0 (resp. $0^{\prime}$ ) and the trivial inequality

$$
\left\|N^{k} \psi\right\| \leqq\left\|N^{l} \psi\right\|, \quad \text { for } k \leqq l,
$$

we obtain

$$
\begin{aligned}
d G_{\lambda}(t) / d t & \leqq 2 k_{3}\left\|N^{n} e^{-i t A_{\lambda}} \psi\right\|^{2} \\
& =2 k_{3} G_{\lambda}(t),
\end{aligned}
$$

for some finite constant $k_{3}$ independent of $\lambda$, which after integration yields the desired Lemma A. $1_{\lambda}$. From this Lemma A.1 follows by essentially the same arguments that gave Lemma 2 as a corollary to Lemma $2_{\lambda}$. Q.E.D.

Remarks. 1) Careful inspection of the proof of Lemma A.1 shows that the conclusions of this lemma remain true if we only assume that

$$
\|\stackrel{(k)}{A} \psi\| \leqq k_{3}\left\|N^{k} \psi\right\|
$$

for some finite constant $k_{3}$ and all $\psi \in D\left(N^{k}\right), k=1,2, \ldots,|n|$.

2) Let $\alpha \in[-|n|,|n|]$; since

$$
N^{2|\alpha|} \leqq N^{2|n|}, \quad \text { on } \quad D\left(N^{|n|}\right) \times D\left(N^{|n|}\right),
$$

Lemma A.1 holds for all $\alpha \in[-|n|,|n|]$ if $A, \dot{A}, \ldots, \stackrel{(|n|)}{A}$ all satisfy the hypotheses of Lemma A.1, (resp. $\stackrel{(k)}{A}$ satisfies (A.3), $k=1, \ldots,|n|$.)

We may now re-formulate Theorems $1_{M}$ and $1_{\infty}$.

Theorem A.2. Let $B$ and $A, \dot{A}, \ldots, \stackrel{(\alpha)}{A}$, (for some $\alpha=1,2,3, \ldots$ ) all satisfy the hypotheses of Theorem 0 .

Let $C_{0}=B$, and

$$
C_{n}=i\left[A, C_{n-1}\right] \text {, weakly on } D\left(N^{\alpha}\right) \times D\left(N^{\alpha}\right),
$$

and

$$
\left\|C_{n} \psi\right\| \leqq K_{2}^{n} n !\left\|N^{\alpha} \psi\right\| ; \quad n=1,2,3, \ldots .
$$

Then

$$
\begin{aligned}
B_{t}= & {\left[\left\{B+\sum_{m=1}^{M-1} \frac{t^{m}}{m !} C_{m}\right.\right.} \\
& \left.\left.+\int_{0}^{t} d t, \ldots, \int_{0}^{t_{M}-1} d t_{M} e^{i t_{M} A} C_{M} e^{-i t_{M} A}\right\} \uparrow D\left(N^{\alpha}\right)\right]^{-}
\end{aligned}
$$

and, for $|t|<K_{2}^{-1}$,

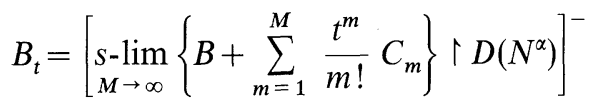

both satisfy

$$
e^{i t A} e^{i s B} e^{-i t A}=e^{i s B_{t}} .
$$


Proof. By Lemma A.1 $D\left(N^{\alpha}\right)=e^{ \pm i t A} D\left(N^{\alpha}\right)$, for all $|t|<\infty . D\left(N^{\alpha}\right)$ is a core for $N$, hence a core for $B$ (by Theorem 0 ). Thus $D\left(N^{\alpha}\right)$ is also a core for $B_{t}$ [defined as in (4.1)]. But on $D\left(N^{\alpha}\right)$

$$
B_{t}=e^{-i t A} B e^{-i t A}
$$

by Lemma A.1.

Applying now Lemma A.1 and the hypotheses of Theorem A.2 (concerning $\left\{C_{n}\right\}_{n=0}^{\infty}$ ), the proof of Theorem A.2 can be completed as in Section 4 .

Acknowledgements. I wish to thank J. Glimm and A. Jaffe for communicating to me the results of [3] prior to publication and $\mathrm{H}$. Araki and B. Simon for very helpful suggestions. This note would not have been written without previous enjoyable collaboration with W. Driessler.

\section{References}

1. Araki,H.: J. Math. Phys. 1, 492 (1960)

2. Driessler,W., Fröhlich,J.: The reconstruction of local observable algebras from the Euclidean Green's functions of a relativistic quantum field theory (to be published)

3. Glimm,J., Jaffe, A.: Proceedings of the 1976 Cargèse Summer School in Theoretical Physics (to appear)

4. Glimm,J., Jaffe, A.: J. Math. Phys. 13, 1568 (1973)

5. Herbst, I. : J. Math. Phys. 17, 1210 (1976)

6. Nelson, E.: J. Funct. Anal. 11, 211 (1972)

7. Nelson,E.: Ann. Math. 70, 572 (1959)

8. Reed, M., Simon, B. : Methods of modern mathematical physics. II. Fourier analysis, self-adjointness. London-New York: Academic Press 1975

9. Simon, B.: The $P(\phi)_{2}$ Euclidean (quantum) field theory. Princeton Series in Physics. Princeton: University Press 1974

10. Simon, J.: Commun. math. Phys. 28, 39 (1972)

11. Streater,R.F., Wightman, A.S.: PCT, spin and statistics and all that. New York: Benjamin 1964

12. Haag, R., Kastler,D.: J. Math. Phys. 5, 848 (1964)

Communicated by H. Araki

Received December 27, 1976

Note Added in Proof: Ed Nelson has informed me that he has derived the conclusions of Theorem 5 under the only assumptions that $A$ and $B$ satisfy the hypotheses of Theorem 0 , and $[A, B]=0$, weakly on $D(N) \times D(N)$. His proof involves showing that $A+i B$ is a normal operator. Subsequently we found a proof of this result based on a straight forward extension of the methods of this paper. Moreover we proved Theorem 3 without the extra-hypothesis on $\dot{X}_{j}$. 